\title{
Avaliação da Cavidade Uterina: Estudo Comparativo entre Histerografia, Histerossonografia e Histeroscopia
}

\author{
Endouterine Cavity Investigation: Comparative Study between X-ray Hysterography, \\ Sonohysterography and Hysteroscopy
}

Rodrigo Coelho Franco, José Cassiano Machado, Jorge Elias Junior, Aderson Tadeu Berezowski Antonio Alberto Nogueira, Maria Matheus de Sala

\section{RESUM0}

\begin{abstract}
Objetivo: realizar um estudo comparativo entre os métodos utilizados para avaliar a cavidade uterina por meio da análise da sensibilidade e especificidade da histerografia e da histerossonografia, comparadas com a histeroscopia (padrão ouro).

Métodos: foi realizado um estudo prospectivo para a investigação da cavidade uterina com a histerografia, a histerossonografia e a histeroscopia em 18 pacientes. Todas as pacientes apresentavam suspeita de anomalias da cavidade uterina. As principais indicações para a investigação foram: distúrbios do ciclo menstrual, sangramento pós-menopausa e alterações observadas em exames ultra-sonográficos sugestivas de pólipos, miomas e/ou espessamento endometrial.

Resultados: a sensibilidade da histerossonografia foi de $90 \%$ e a especificidade $87,5 \%$, quando comparadas à histeroscopia. O valor preditivo positivo foi de $90 \%$ e o negativo $87,5 \%$. A histerografia, por sua vez, apresentou sensibilidade de $70 \%$, especificidade de $75 \%$, valor preditivo positivo de $77,7 \%$ e valor preditivo negativo de $75 \%$.

Conclusão: a histerossonografia parece ser um bom método alternativo para a avaliação da cavidade uterina, identificando algumas lesões não observadas à ultra-sonografia convencional ou à histerografia, e permitindo a triagem das pacientes candidatas à realização de uma histeroscopia diagnóstica ou cirúrgica. A histerografia apresentou resultados inferiores à histerossonografia, e não parece ser de utilidade na triagem das pacientes.
\end{abstract}

PALAVRAS-CHAVE: Histerossonografia. Histerografia. Endométrio. Pólipo endometrial. Histeroscopia.

\section{Introdução}

São diversas as causas de sangramento vaginal proveniente do útero. Elas podem ocorrer em qualquer idade, sendo resultado de problemas relacionados à anovulação, lesões anatômicas benignas ou malignas, gravidez ou distúrbios hormonais. Numa tentativa de se diagnosticar a causa do sangramento, pode-se lan-

Setor de Reprodução Humana do Departamento de Ginecologia e Obstetrícia da Faculdade de Medicina de Ribeirão Preto da Universidade de São Paulo.

Av. Bandeirantes, 3900 - Monte Alegre - Ribeirão Preto - SP CEP 14049-900.

Correspondência:

Maria Matheus de Sala

Rua Rui Barbosa, 521 - apto 112

14015-120 - Ribeirão Preto - SP çar mão de exames como a biópsia endometrial, a curetagem uterina, a ultra-sonografia transvaginal ou abdominal, a histerossalpingografia e, idealmente, a histeroscopia.

A histerossalpingografia é um exame aceito há várias décadas para avaliação da cavidade uterina e permeabilidade tubária. É um método clássico no diagnóstico de anormalidades uterinas $^{1,2}$. É, juntamente com a cromotubagem laparoscópica, o "padrão ouro" para avaliação da permeabilidade tubária ${ }^{3}$. A laparoscopia é considerada atualmente um exame confiável para o diagnóstico de causas tubárias de infertilidade, permitindo visualizar diretamente as anormalidades morfológicas das trompas e coletar material para exames subsidiários ${ }^{4}$. Quando se comparam a histerossalpingografia e a laparoscopia 
deve-se ter em mente que ambos os procedimentos se completam.

Durante a sua evolução ocorreram modificações nas técnicas, principalmente relacionadas aos equipamentos e contrastes utilizados. Heikkinen et al. ${ }^{5}$ referem que a histerossalpingografia está associada a riscos como a irradiação dos órgãos da pelve, infecção intra-uterina e abdominal e dor, os quais podem ser evitados tomando-se as devidas precauções.

Salle et al. ${ }^{2}$ opinam ser praticamente impossivel detectar alterações de fusão dos ductos de Müller ao exame histerossalpingográfico. Na detecção destas anormalidades, este exame tem baixa especificidade com uma taxa de falso-negativo em torno de $10 \%{ }^{6}$. A localização de pólipos mas paredes anterior ou posterior, bem como a identificação de sua base de inserção e o diagnóstico diferencial com mioma submucoso ficam prejudicados ${ }^{7}$.

A ultra-sonografia transvaginal é essencial para a avaliação das causas de sangramento pósmenopausa, controle do endométrio na reposição hormonal e qualquer alteração na cavidade pélvica. Porém, não permite a diferenciação inequívoca das imagens endometriais entre pólipos e hiperplasias, malformações uterinas e fenômenos proliferativos resultantes de terapia de reposição hormonal ${ }^{8}$, assim como na determinação da localização submucosa ou intramural de um mioma ${ }^{9}$.

Para uma melhor avaliação endometrial, Parsons e Lense $^{10}$ descreveram a realização de ecografia transvaginal simultaneamente à introdução de uma solução salina na cavidade uterina. Ainda hoje, alguns autores recomendam a infusão de solução salina como agente de primeira escolha para a investigação da cavidade endometrial ${ }^{11}$.

A instilação de solução salina na cavidade uterina, simultaneamente ao exame ecográfico abdominal, já havia sido relatada anteriormente com alguns resultados pouco satisfatórios ${ }^{12}$. Os inconvenientes ocorriam, principalmente, nas pacientes obesas, nas quais a distância entre o transdutor e o órgão estava aumentada, prejudicando a qualidade da imagem. Outra desvantagem era a necessidade de as pacientes realizarem o exame com a bexiga cheia, pré-requisito do exame abdominal, para melhorar a qualidade das imagens.

Esse método procura, na verdade, uma avaliação fidedigna com baixo custo, pouco desconforto e mínimos efeitos colaterais. Busca-se uma alta sensibilidade e especificidade quando se confronta com a histeroscopia, exame de escolha para avaliação da cavidade uterina. No entanto, a instilação de soluções dentro da cavidade uterina é contra-indicada na presença de hematometra, suspeita de gestação, sangramento vaginal e doença inflamatória pélvica.

A histeroscopia é um excelente método para a avaliação da cavidade uterina, seja em casos de sangramento indeterminado ou para a investigação de pacientes inférteis. Devido ao alto custo do equipamento e à falta de treinamento especializado, somente uma pequena percentagem de ginecologistas estão aptos para a realização do exame em consultório. A histeroscopia ambulatorial, entretanto, é importante porque detecta e localiza lesões anatômicas como pólipos, miomas, hiperplasias e carcinomas, e permite a realização de biópsia dirigida.

O objetivo deste trabalho foi realizar um estudo comparativo entre a histerografia, histerossonografia e histeroscopia, realizadas no Hospital das Clínicas da Faculdade de Medicina de Ribeirão Preto da Universidade de São Paulo, como métodos de avaliação da cavidade uterina.

\section{Pacientes e Métodos}

Foi realizado um estudo prospectivo com 18 pacientes que apresentavam suspeita de alteração da cavidade uterina, mediante a utilização de histerografia, histerossonografia e histeroscopia. As indicações para a investigação foram distúrbios do ciclo menstrual, sangramento pós-menopausa e alterações observadas em exames ultra-sonográficos (pólipos, miomas, espessamento endometrial). Inicialmente, as pacientes foram submetidas a histerografia ou a histerossonografia, aleatoriamente, sendo posteriormente realizada a histeroscopia.

A idade das pacientes variou de 33 a 60 anos, com mediana de 45. Quatro pacientes eram menopausadas e 14 ainda menstruavam. Onze pacientes já haviam sido submetidas a laqueadura tubária previamente. Somente uma paciente que se encontrava em idade fértil não era laqueada. Nas pacientes laqueadas ou menopausadas os exames foram realizados sem a preocupação com o dia do ciclo. Na que se encontrava em idade fértil e sem proteção contra concepção, os exames foram realizados após o término da menstruação.

As histerografias foram realizadas no Departamento de Radiologia do HCFMRP-USP, com contraste hidrossolúvel e após uma ultra-sonografia de controle. Para a introdução de contraste foi utilizado um histerossalpingógrafo de metal. 
A histeroscopia e a histerossonografia foram realizadas no Departamento de Ginecologia e Obstetrícia do HCFMRP-USP. Para a histeroscopia utilizou-se o microhisteroscópio de Hamou I e II, marca Storz, com óptica de 4,0 mm de diâmetro, com ângulo de inclinação de $30^{\circ}$ e camisa de $5,0 \mathrm{~mm}$, com canal para passagem de meio distensor. Para a distensão da cavidade uterina utilizou-se meio gasoso $\left(\mathrm{CO}_{2}\right)$ com controle eletrônico de fluxo e pressão, insuflado com o Microhisteroflator de Hamou, Storz 26431020.

A histerossonografia foi realizada com um aparelho da marca ATL (Advanced Technology Laboratories) modelo HDI 3.000, com sonda vaginal de multifreqüência de 5 a $9 \mathrm{mHz}$. Para a introdução da solução salina, foi utilizada uma sonda de $2 \mathrm{~mm}$ de diâmetro, modelo ZUI 2.0 (Zinnantti Surgical Instruments, Chatsworth, CA), com balão inflável em sua extremidade.

Na histerossonografia, após a introdução de um espéculo vaginal, era realizada uma limpeza local com soro fisiológico e anestesia local com lidocaína spray a $10 \%$. Em alguns casos houve a necessidade de pinçamento do colo uterino, utilizando-se a pinça de Pozzi. A seguir, era introduzida a sonda até ultrapassar o orifício interno do colo uterino, inflando-se o balão da extremidade quando possível. Retirado o espéculo, era introduzido o transdutor transvaginal e injetado o soro fisiológico na cavidade uterina. O exame foi realizado sempre pelos mesmos profissionais.

As 3 modalidades de exames foram realizadas por grupos de profissionais que não tinham conhecimento dos resultados obtidos com as outras metodologias.

Em todas as pacientes colheu-se citologia cervical uterina e sorologia para hepatite B e HIV previamente à realização dos exames.

Nos casos em que era indicado, foi realizado o exame histopatológico de material retirado por biópsia endometrial ou por histeroscopia cirúrgica.

Os resultados foram analisados com o auxílio do software Graph Pad Prism, Version 2.00.

Este estudo foi aprovado pelo Comitê de Ética Médica do Hospital das Clínicas da Faculdade de Medicina de Ribeirão Preto e as pacientes assinaram um termo de consentimento pósinformação.

\section{Resultados}

A sensibilidade da histerossonografia foi de $90 \%$ e a especificidade $87,5 \%$, quando compara- da à histeroscopia. O valor preditivo positivo foi de $90 \%$ e o negativo $87,5 \%$. Em relação à histerografia encontramos uma sensibilidade de $70 \%$, especificidade de $75 \%$, valor preditivo positivo de $77,7 \%$ e valor preditivo negativo de $75 \%$ (Figura 1).

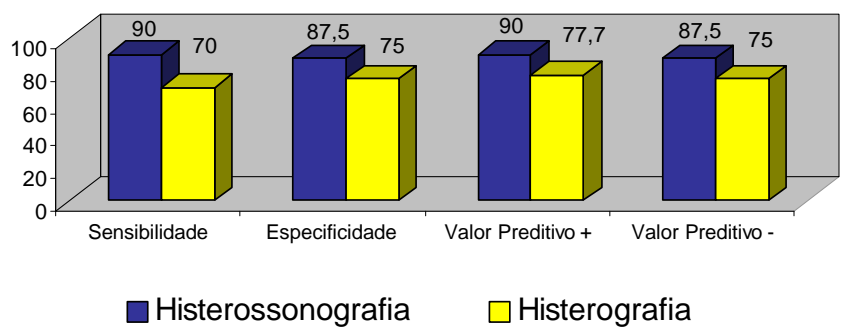

Figura 1 - Valores de sensibilidade e especificidade da histerossonografia e histerografia, quando comparadas com a histeroscopia.

Os achados histeroscópicos revelaram pólipo endometrial, mioma submucoso, endométrio disfuncional, pseudosinéquias e exames sem alteração, incluindo a atrofia endometrial. Os achados histerossonográficos e histerográficos se encontram respectivamente nas Figuras 2 e 3.

\section{Achados Histerossonográficos}

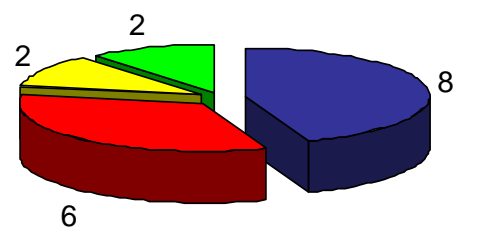

Normal

$\square$ Pólipo

$\square$ Mioma + Pólipo

Espessamento endometrial

Figura 2 - Distribuição dos achados obtidos pela histerossonografia.

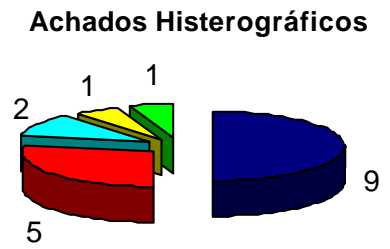

Normal

Pólipo

$\square$ Mioma + Pólipo

Imagem especulada

Deformidade endometrial

Figura 3 - Distribuição dos achados obtidos pela histerografia.

O exame histopatológico realizado em oito pacientes com indicação de biópsia mostrou a presença de 4 casos de endométrio disfuncional e 4 casos de pólipos endometriais. Em um outro caso, a paciente realizou acompanhamento fora do Serviço. 
A comparação dos achados histerossonográficos com os histeroscópicos mostra uma associação estatisticamente significante $(p=0,0029$; teste exato de Fisher).

A comparação dos achados histerográficos com os histeroscópicos não mostra uma associação estatisticamente significante $(\mathrm{p}=0,1534$; teste exato de Fisher), embora o número de casos seja pequeno para conclusão estatisticamente significativa $(\beta=50 \%)$.

Em 3 casos a sonda não ultrapassou o orifício interno do colo e foi inflada dentro do canal endocervical.

Nos casos em que a paciente referia muita dor durante o exame, o balão da sonda era mantido vazio, diminuindo assim, a pressão intrauterina e, conseqüentemente, a dor. Porém, ocorria a desvantagem de fluxo retrógrado acentuado do soro fisiológico, observado com maior freqüência nas pacientes laqueadas.

$\mathrm{O}$ volume de soro fisiológico introduzido para avaliar a cavidade uterina variou de 5 a 20 ml. O maior volume era utilizado nos casos em que o balão da sonda não era inflado.

Após a realização da histerossonografia, em alguns casos, ocorreu sangramento vaginal discreto, atribuído à descamação do endométrio distendido pelo soro fisiológico.

\section{Discussão}

Os trabalhos que estudaram os métodos diagnósticos aqui analisados mostram a alta sensibilidade e especificidade da histerossonografia quando comparada com a histeroscopia. Mesmo trabalhos com pequeno número de casos mostram resultados similares (Tabela 1). Os dados e resultados não devem ser diretamente comparados, pelo fato de serem estudos diferentes com grupos diferentes de pacientes (prospectivos ou retrospectivos; inclusão de pacientes com infertilidade ou menopausadas, etc).

Tabela 1 - Dados da literatura sobre a sensibilidade e especificidade da histerossonografia e da histerografia em relação à histeroscopia.

\begin{tabular}{|c|c|c|c|c|c|}
\hline \multirow[t]{2}{*}{ Autor } & \multirow[t]{2}{*}{ Pacientes } & \multicolumn{2}{|c|}{ Histerossonografia } & \multicolumn{2}{|c|}{ Histerografia } \\
\hline & & Sensibilidade (\%) & Especificidade (\%) & Sensibilidade (\%) & Especificidade (\%) \\
\hline Randolph et al., $1986^{12}$ & 61 & 98 & 100 & 98 & 92 \\
\hline Bonilla-Mussoles, $1992^{8}$ & 22 & 87 & 100 & 100 & 85 \\
\hline Reis et al., $1996^{22}$ & 36 & 90,9 & 92,3 & 64,3 & 75 \\
\hline Widrich et al., $1996^{18}$ & 130 & 96 & 88 & - & - \\
\hline Ayida et al., $1997^{23}$ & 38 & 87,5 & 100 & - & - \\
\hline Keltz et al., $1997^{24}$ & 18 & 100 & 100 & 90 & 20 \\
\hline Williams et al., $1998^{13}$ & 39 & 100 & 85 & - & - \\
\hline Gronlund et al., $1999^{9}$ & 60 & 90,9 & 100 & - & - \\
\hline Presente trabalho, 2000 & 18 & 90 & 87,5 & 70 & 75 \\
\hline
\end{tabular}

No presente estudo foram utilizadas pacientes que apresentavam algum tipo de alteração endometrial, havendo a possibilidade de essa alteração tratar-se de neoplasia. Uma preocupação constante no início do trabalho foi a possibilidade de o soro fisiológico introduzido na cavidade uterina carrear células neoplásicas através das tubas uterinas para o abdome, provocando assim uma metastatização iatrogênica. Para tanto, procurava-se explorar somente a cavidade uterina, evitando a passagem da solução salina pelas trompas, exercendo uma menor pressão durante a instilação do soro fisiológico. De fato, Gronlund et al. ${ }^{9}$ relataram que uma lenta e fraca pressão de infusão diminui muito o risco do transporte celular e, portanto, o risco não seria diferente do da histerografia. Entretanto, alguns autores sugerem que, quando há suspeita de neoplasia ou hiperplasia endometrial, deve-se realizar uma biópsia dirigida previamente ao exame ${ }^{13}$. É importante lembrar que a curetagem aspirativa é falha em alguns casos, principalmente para pólipos ${ }^{7}$, mas que segundo Maia Júnior et al. ${ }^{14}$ a sua sensibilidade está relacionada com o diâmetro da cureta, sendo os melhores resultados obtidos com curetas com diâmetro maior de $5 \mathrm{~mm}$.

Existem vários estudos na literatura em que se realizou a histerografia em pacientes com câncer de endométrio já diagnosticado, com a finalidade de precisar o volume do tumor e sua localização. Sala et al. ${ }^{15}$ realizaram a histerografia em 
pacientes com câncer endometrial prévio ao tratamento radioterápico e concluíram que as imagens obtidas permitem um planejamento adequado para o tratamento individualizado, ajudando a determinar o volume do tumor, localização e extensão dentro da cavidade uterina.

Nagele et al. ${ }^{16}$ mostraram a possibilidade de se encontrarem células endometriais e tubárias na cavidade abdominal de 1/4 das pacientes que realizaram a histeroscopia com dióxido de carbono ou solução salina sob uma pressão intra-uterina de 100 a $150 \mathrm{mmHg}$. Concluíram, portanto, que a histeroscopia com o dióxido de carbono pode por si só carrear células para a cavidade abdominal.

Muitos autores não acreditam na possibilidade de metastatização e propõem a histerossonografia para investigação da cavidade uterina dessas pacientes, sugerindo a necessidade de maiores estudos. Outros sugerem evitar a injeção de contraste utilizando altas pressões e, principalmente, usar como meio de contraste água destilada estéril, para provocar a lise de células descamadas. Deve-se evitar a instilação de fluidos enquanto há sangramento menstrual ativo, a fim de não carrear detritos para dentro da cavidade peritoneal, com possivel semeadura de implantes endometriais ectópicos.

No presente estudo não foi identificado líquido livre no fundo de saco de Douglas após o exame $(61,1 \%$ das pacientes eram laqueadas), permitindo afirmar que a pressão de injeção do líquido não ultrapassou os valores de 6 a 7 $\mathrm{mmHg}$.

A histerossonografia praticamente não apresenta complicações. Neste estudo houve um caso apenas em que a paciente apresentou episódio curto e transitório de hipotensão e tontura, provavelmente secundário e atribuído a um efeito vagal, relatado por Goldstein ${ }^{17}$. Não se observaram outros tipos de complicações. No presente estudo não se realizou nenhum tipo de medicação prévia aos exames, uma vez que as pacientes não apresentavam sinais ou sintomas de infecção do trato genital inferior. Alguns autores fazem uso de antiinflamatório não-hormonal previamente ao exame ${ }^{18}$, outros após ${ }^{1}$, ou ainda, de antibióticoprofilaxia ${ }^{5}$, principalmente em pacientes com prolapso de valva mitral. Guando necessário a paciente deve ser medicada para suavizar a dor, sem suprimi-la totalmente, permitindo que a mesma proporcione informações importantes principalmente sobre aumento exagerado de pressão dentro das tubas, evitando-se lesões iatrogênicas.

São referidos na literatura casos em que não é possível passar a sonda pelo canal endocervical. Há relatos de uso de pastas e geléias à base de misoprostol aplicadas no orifício externo do colo uterino na véspera do exame, possibilitando uma melhor permeabilidade do canal endocervical para a passagem da cânula ${ }^{19}$.

Em alguns casos, por dificuldade em se transpor o orifício interno ou mesmo para não atrapalhar a visualização do segmento inferior do útero, o balão da sonda pode ser inflado dentro do canal endocervical ${ }^{6,19}$. Não foi encontrada na literatura a incidência do insucesso na introdução da sonda pelo colo uterino. Em relação à histeroscopia essa falha ocorre em $4,1 \%$ dos casos, as quais são devidas a problemas de obstrução do canal ou de dor durante a passagem da sonda, incluindo pacientes com e sem anestesia ${ }^{6}$. A comparação não é válida com a histerossonografia pois o calibre da sonda uterina é bem menor que o de uma óptica histeroscópica.

Alguns autores costumam não inflar o balão e Salle et al. ${ }^{2}$ utilizaram cateter que não continha o balão na extremidade. Quando não se tem o cateter específico para os exames, ou mesmo quando se pretende diminuir os custos, é possível a realização com sonda Foley de pequeno calibre ou idealmente a sonda de Nelaton ${ }^{20}$.

Apesar de a histeroscopia ser o exame de escolha para a avaliação da cavidade uterina, Cicinelli et al. ${ }^{21}$ relataram que a ultra-sonografia transvaginal é mais precisa que a histeroscopia para o mapeamento e mensuração de miomas submucosos, embora freqüentemente não consiga distinguí-los de pólipos. Com a histerossonografia, tais dificuldades ficam diminuídas, permitindo o diagnóstico diferencial de tais patologias da cavidade uterina.

Embora hoje se disponha de avançados métodos para a avaliação de patologias ginecológicas, nenhum dos exames por si só pode explorar completamente todas as alterações do trato genital feminino. Cada técnica tem suas vantagens, limites, riscos e falsos resultados. É importante ressaltar que os três métodos se complementam na avaliação das alterações da cavidade uterina.

Concluimos que a histerossonografia parece ser um bom método alternativo para a avaliação da cavidade uterina, identificando algumas lesões não-observadas à ultra-sonografia convencional ou à histerografia, permitindo a triagem das pacientes candidatas à realização de uma histeroscopia diagnóstica ou cirúrgica.

A histerografia apresentou resultados inferiores à histerossonografia, e não parece ser de utilidade na triagem das pacientes. 


\section{SUMMARY}

Purpose: to compare the methods used to investigate the endouterine cavity by testing the sensitivity and specificity of X-ray hysterography and sonohysterography compared with hysteroscopy (gold standard).

Methods: we carried out a prospective study with 18 patients who, due to symptoms such as irregular menstrual cycles, unexplained postmenopausal uterine bleeding and ultrasound disturbance, were candidates for uterine cavity investigation by X-ray hysterography, sonohysterography and hysteroscopy.

Results: sonohysterography sensitivity and specificity were 90 and $87.5 \%$, respectively. Positive and negative predictive values were 90 and $87.5 \%$. For X-ray hysterography, sensitivity, specificity, positive and negative predictive values were 70, 75, 77.7 and $75 \%$, respectively.

Conclusion: the use of saline instilation into the endometrial cavity in order to enhance the acuracy of the vaginal ultrasonography seems reliable as a mean to distinguish lesions in the uterine cavity, thereby facilitating the identification of candidates for diagnostic or operative hysteroscopy. X-ray hysterography produces results inferior to hysterosonography.

KEY WORDS: Sonohysterography. Hysterography. Endometrium. Endometrial polyp. Hysteroscopy.

\section{Referências}

1. Ayida G, Kennedy S, Barlow D, Chamberlain P. A comparison of patient tolerance of hysterosalpingo-contrast sonography (HyCoSy) with Echovist ${ }^{\circ}-200$ and $\mathrm{X}$-ray hysterosalpingography for outpatient investigation of infertile women. Ultrasound Obstet Gynecol 1996; 7:201-4.

2. Salle B, Sergeant P, Gaucherand P, Guimont I, de Saint Hilaire P, Rudigoz RC. Transvaginal hysterosonographic evaluation of septate uteri: a preliminary report. Hum Reprod 1996; 11:1004-7.

3. Schlief R, Deichert U. Hysterosalpingo-contrast sonography of the uterus and fallopian tubes: results of a clinical trial of a new contrast medium in 120 patients. Radiology 1991; 178:213-5.

4. Mol BW, Collins JA, Burrows EA, van der Veen F, Bossuyt PM. Comparison of hysterosalpingography and laparoscopy in predicting fertility outcome. Hum Reprod 1999; $14: 1237-42$.

5. Heikkinen H, Tekay A, Volpi E, Martikainen H, Jouppila P. Transvaginal salpingosonography for the assessment of tubal patency in infertile women: methodological and clinical experiences. Fertil Steril 1995; 64:293-8.

6. Kim AH, McKay H, Keltz MD, Nelson PH, Adamsom GD. Sonohysterographic screening before in vitro fertilization. Fertil Steril 1998; 69:841-4.

7. Syrop CH, Sahakian V. Transvaginal sonographic detection of endometrial polyps with fluid contrast augmentation. Obstet Gynecol 1992; 79:1041-3.

8. Bonilla-Musoles F, Raga F, Osborne NG, Blanes J, Ceelho F. Three-dimensional hysterosonography for the study of endometrial tumors: comparison with conventional transvaginal sonography, hysterosalpingography, and hysteroscopy. Gynecol Oncol 1997; 65:245-52.

9. Gronlund L, Hertz J, Helm P, Colov NP. Transvaginal sonohysterography and hysteroscopy in the evaluation of female infertility, habitual abortion or metrorrhagia. A comparative study. Acta Obstet Gynecol Scand 1999; 78:415-8.

10.Parsons AK, Lense JJ. Sonohysterography for endometrial abnormalities: preliminary results. J Clin Ultrasound 1993; 21:87-95.

11.Hamilton JA, Larson AJ, Lower AM, Hasnain S, Grudzinskas JG. Evaluation of the performance of hysterosalpingocontrast sonography in 500 consecutive, unselected, infertile women. Hum Reprod 1998; 13:1519-26.

12.Randolph JF Jr, Ying YK, Maier DB, Schmidt CL, Riddick DH. Comparison of real time ultrasonography, hysterosalpingography and laparoscopy/hysteroscopy in the evaluation of uterine abnormalities and tubal patency. Fertil Steril 1986; 46:828-32.

13.Williams CD, Marshburn PB. A prospective study of transvaginal hydrosonography in the evaluation of abnormal uterine bleeding. Am J Obstet Gynecol 1998; 179:292-8.

14. Maia Júnior H, Maltez A, Calmon LC, Marques D, Oliveira M, Coutinho E. Comparison between suction curettage, transvaginal sonography and hysteroscopy for the diagnosis of endometrial polyp. Gynecol Endosc 1998; 7:127-32.

15.Sala MM, Sala MA, Franceschini SA, Baruffi I. Histerografia no carcinoma de endométrio. Rev Imagem 1986; 8:79-82.

16.Nagele F, Wieser F, Deery A, Hart R, Magos A. Endometrial cell dissemination at diagnostic hysteroscopy: a prospective randomized crossover comparison of normal saline and carbon dioxide uterine distension. Hum Reprod 1999; 14:2739-42. 
17.Goldstein SR. Use of ultrasonohysterography for triage of perimenopausal patients with unexplained uterine bleeding. Am J Obstet Gynecol 1994; 170:565-70.

18. Widrich T, Bradley LD, Mitchinson AR, Collins RL. Comparison of saline infusion sonography with office hysteroscopy for the evaluation of the endometrium. Am J Obstet Gynecol 1996; 174:1327-34.

19.Pacheco GHAS, Shibasaki WS, Pacheco Filho MF. Avaliação ultra-sonográfica da cavidade uterina e permeabilidade tubária. Rev Bras Ultrason 1997; 4:15-21.

20.Kremer C, Barik S, Duffy S. Flexible outpatient hysteroscopy without anaesthesia: a safe, successful and well tolerated procedure. $\mathrm{Br} \mathrm{J}$ Obstet Gynaecol 1998; 105:672-6.
21.Cicinelli E, Romano F, Anastasio PS, Blasi N, Parisi C, Galantino P. Transabdominal sonohysterography, transvaginal sonography, and hysteroscopy in the evaluation of submucous myomas. Obstet Gynecol 1995; 85:42-7.

22.Reis MMBB, Soares SR, Miranda SP, Camargos AF. Avaliação da cavidade uterina pela histerossonografia. Reprod Clim 1997; 12:19-23.

23.Ayida G, Chamberlain P, Barlow D, Kennedy S. Uterine cavity assessment prior to in vitro fertilization: comparison of transvaginal scanning, saline contrast hysterosonography and hysteroscopy. Ultrasound Obstet Gynecol 1997; 10:59-62.

24.Keltz MD, Olive DL, Kim AH, Arici A. Sonohysterography for screening in recurrent pregnancy loss. Fertil Steril 1997; 67:670-4.

\section{Recadastramento de Sócios}

A partir do próximo mês, a FEBRASGO estará dando início ao processo de recadastramento dos associados das federadas.

Como sabemos, todo cadastro é dinâmico - muda todo momento e algumas vezes nos esquecemos de comunicar as eventuais mudanças às nossas federadas. Com isto criamos problemas de comunicação entre nós e nossa federada/FEBRASGO - recebimento de Femina e RBGO, Jornal da FEBRASGO e Manuais de Orientação - bem como informações sobre cursos e concursos - TEGO e Áreas de Atuação, Congressos, Carteiras de sócios etc.

Para aqueles que têm acesso à internet, o formulário de recadastramento está disponibilizado no site da FEBRASGO www.febrasgo.org.br - podendo ser preenchido e remetido por e-mail (vide link no final do formulário)

Colega, recadastre-se, esta é a única forma de nos comunicarmos com Você.

A Diretoria 